\title{
Mesoporous Silicon with Nanostructured Au-Ti Front-side Electrical Contact as Efficient Capacitive Sensor Toward Different Alcohols in Liquid Phase
}

\author{
F. A. Harraz ${ }^{1,2^{*}}$, M. A. Rashed ${ }^{1,3}$, A. S. Alkorbi ${ }^{4}$, M. Faisal ${ }^{1,5}$
}

${ }^{1}$ Promising Centre for Sensors and Electronic Devices (PCSED), Advanced Materials and Nano-Research Centre, Najran University, P.O. Box: 1988, Najran 11001, Saudi Arabia

${ }^{2}$ Nanomaterials and Nanotechnology Department, Central Metallurgical Research and Development Institute (CMRDI), P.O. 87 Helwan, Cairo 11421, Egypt

${ }^{3}$ Department of Chemistry, Faculty of Science, Mawlana Bhashani Science and Technology University, Santosh, Tangail 1902, Bangladesh ${ }^{4}$ Department of Chemistry, Faculty of Science and Arts at Sharurah, Najran University, Saudi Arabia

${ }^{5}$ Department of Chemistry, Faculty of Science and Arts, Najran University, Saudi Arabia

*Corresponding author: E-mail: faharraz@nu.edu.sa

Received 1 November 2021

Revised 10 November 2021

Accepted for publication 11 November 2021

Published online 11 November 2021

\section{Abstract}

A novel capacitive sensor using mesoporous silicon (PSi) layer with a front electrical contact composed of Au-Ti nanostructure for sensitive detection of a group of alcohols in the liquid phase is designed and demonstrated. The PSi layer was firstly synthesized by the photo-electrochemical anodization of single crystalline $n$-type Si wafer in hydrofluoric acid based etching solution. The electrical contact was smoothly formed onto the front surface (directly exposed to the porous film) using Au-Ti nanocomposite by photolithographic technique and thermal evaporation approach. The as-fabricated PSi-Au-Ti electrical sensor exhibits efficient sensing response with a reversible behavior during the direct exposure and infiltration with liquid methanol and ethanol. However, the response toward propanol and butanol was less efficient and irreversible. The newlydeveloped electrical sensor was found efficient and promising also for the detection of pure water, which is advantageous for the future sensing ability of aqueous solutions. The observed change in capacitance during the sensing response is related to the generation of a local electrical field at the PSi interface, which led to a shift in the energy level of surface states, generating a modified space-charge region and finally affected the energy distribution and capacitance change.

\section{Graphical Abstract}

\section{Meso-porous silicon}

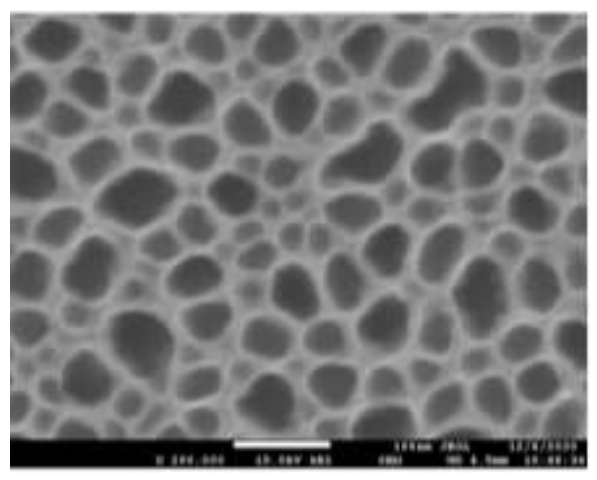

\section{Real-time capacitance response}

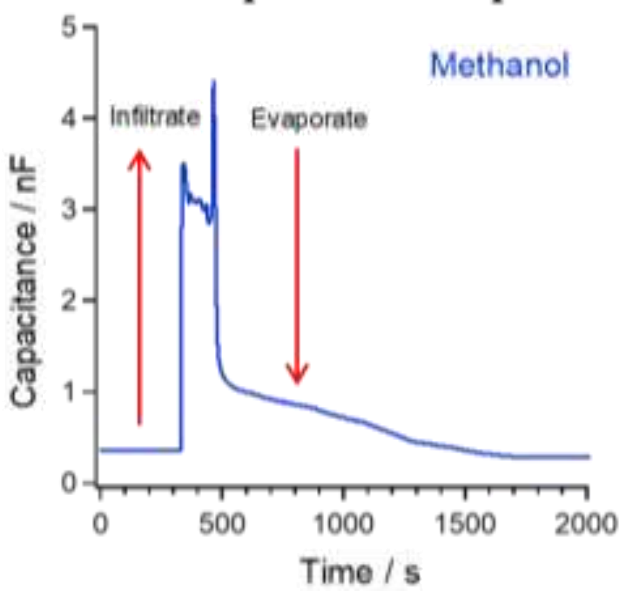

Keywords: Photo-electrochemical etching; Porous silicon; Photolithography; Electrical sensor; Capacitance. 


\section{Introduction}

Porous silicon (PSi) has been first observed accidentally by Arthur and Ingeborg Uhlir in 1956 at the Bell Labs during the electrochemical polishing of both germanium and silicon wafers [1]. During the electrochemical anodization to reach the polishing regime, they have observed deposits on the surface of the silicon wafer with different colors, mainly black, brown, or red deposits. Those silicon deposits were porous in nature; however, they did not realize at that time the porous nature and therefore they have proposed that the deposits consisted of silicon suboxide without further clarification. Various fabrication techniques have been established to smoothly synthesize PSi layers or films, including physical and chemical processes, physicochemical and electrochemical methodologies [2,3]. Now, it is generally accepted that the electrochemical route is the most straightforward and proper technique to obtain homogeneous, well-distributed porous films with chemically stable hydrogen-passivated silicon surfaces [4,5]. The synthesis of PSi films by the electrochemical anodization could be carried out either via applying the galvanostatic or the potentiostatic technique, nevertheless, the synthesis under the constant current (galvanostatic mode) is highly preferable because perfect control of the film porosity could be achieved [6]. Particularly, the fabricated PSi films/layers via the galvanostatic approach are often produced by the electrochemical etching of silicon in hydrofluoric acid-based electrolyte mixed with some surfactant like ethanol, and the starting silicon wafer is a single crystalline substrate, which would lead to the formation of various pore arrays with nano/microporous silicon features [7-12]. Such a wide range of pore sizes and morphologies was reported to be dependent greatly on the electrolyte concentration of fluoride ions, solution $\mathrm{pH}$, the applied anodic current intensity, the use of organic additives, operating temperature, the silicon wafer characteristics ( $p$-type or $n$-type silicon with crustal orientation) and whether the electrochemical anodization is being conducted in the dark or under the illumination $[13,14]$. In addition to this, the etching time was found to be the main factor to control and determine the produced pore length (the thickness of PSi layer).

The main characteristics of the PSi that led to increased interest for technological applications are the huge surface-to-volume ratio of the porous layer, together with its high tendency towards oxidation. In the literature, one can find a wide range of potential application of PSi layers, including silicon-oninsulator (SOI) technology $[15,16]$, active sensing film in chemical sensors [17-20], in micromachining $[21,22]$, in photovoltaics and biomedical related fields $[23,24]$ and many other fields. Regarding the sensing application of PSi film, the motivation behind its use in this promising field is mainly related to the high degree of surface reactivity and the open porous structure with huge surface area. Furthermore, the pore size and its morphology play also a deceive role in sensing application because the morphology, as well as the pore diameter, can control/limit targeting species that can be adsorbed and smoothly infiltrated into the pores during the sensing event. Especially for the electrical sensors based on PSi films, porous structures are firstly produced onto silicon wafer substrate, followed by creating good electrical contacts to PSi surface, either onto the front, top surface or at the rear face, backside of the silicon [2528]. Examples of this type of PSi electrical sensors include the synthesis and operation of a PSi gas sensor following the current changes induced due to the change of the dipole moment of examined gas [29]. A humidity sensor using PSi-related structures has been also demonstrated based on the variation of recorded current with humidity [30]. In comparison to the commercially available metal oxide chemical sensors, the main obstacles facing the chemical sensors based on semiconductor metal oxides are the lack of selectivity, the sensor device must be operated at different temperatures, and the lack of compatibility with the existing silicon technologies. Therefore, it can be realized that PSi layer is a unique active material and considered as a perfect platform in sensing applications as it can be operated at room temperature and can be employed/implemented on the same substrate of the existing silicon technology.

Great efforts have been done worldwide with the purpose to develop efficient electrical sensors using different active nanomaterials to detect organic molecules. In the light of the above explanation, the PSi is considered as one of the promising and outstanding sensing materials owing to its ease of fabrication, large produced surface area, possibility to tune the pore size and morphology [3,4,12,25]. However, the success to design and fabricate an efficient electrical contact with the PSi surface is still a challenge and is considered as a wide room for further research and development. In the current study, novel front side electrical contact of $\mathrm{Au}-\mathrm{Ti}$ nanocomposite fabricated by the photolithography 
and lift-off processes onto the electrochemically synthesized PSi film is designed and fabricated. The newly developed PSi nanostructure with the top electrical contact is then applied, utilized successfully as an efficient electrical (capacitive) sensor for the sensitive detection of a group of alcohols in the liquid phase including methanol, ethanol, propanol, and butanol. The PSi film fabrication and characterization, creation of electrical contact, and the detection of the different alcohols are addressed and thoroughly discussed.

\section{Experimental}

\subsection{Photo-electrochemical synthesis of}

\section{mesoporous silicon}

The starting silicon wafer used in this study has the following characteristics: $n$-type $\mathrm{Si}$ (100) polished from one side with a resistivity of $0.010-0.018 \Omega \mathrm{cm}$. Before starting the photo-electrochemical anodization, the Si wafer was immersed in $5 \mathrm{wt} \%$ aq. hydrofluoric acid (HF) for a couple of minutes to dissolve any native oxides that remain on the surface, followed by dipping the wafer in acetone for another few minutes, and then left for natural drying. The photo-electrochemical anodization was conducted by applying the galvanostatic mode using an etching solution composed of $28 \mathrm{wt} . \% \mathrm{HF} / \mathrm{H}_{2} \mathrm{O} / \mathrm{EtOH}$, in a two-electrode system of which a Pt rod acts as a counter electrode while the silicon wafer acts as the working electrode at anodic current density of 50 $\mathrm{mA} / \mathrm{cm}^{2}$ for 75 seconds. After the anodization, the silicon wafer specimen with porous layer is carefully taken from the cell and dried under the flow of nitrogen gas. It is worthy to mention here that for the electrochemical etching to proceed, the $n$-type $\mathrm{Si}$ wafer has been illuminated during the anodization process to generate necessary holes for the oxidation and dissolution of silicon. The applied anodic current and consequently the generated potential should locate within the Si pore formation regime, to avoid working in the electro-polishing regime (higher potential value region). The photo-electrochemical anodization of $\mathrm{Si}$ in $\mathrm{HF}$ electrolytic solution could proceed via a two-electron oxidation reaction according to equations (1-3) [31,32]:

Electrochemical step:

$\mathrm{Si}+2 \mathrm{~F}^{-}+2 h^{+} \longrightarrow\left[\mathrm{SiF}_{2}\right]$

Chemical step:

$\left[\mathrm{SiF}_{2}\right]+4 \mathrm{~F}^{-}+2 \mathrm{H}^{+} \longrightarrow \mathrm{SiF}_{6}^{2-}+\mathrm{H}_{2}$

Net reaction:
$\mathrm{Si}+6 \mathrm{~F}^{-}+2 \mathrm{H}^{+}+2 h^{+} \longrightarrow \mathrm{SiF}_{6}^{2-}+\mathrm{H}_{2}$

\subsection{Fabrication of front-side electrical contact}

\section{by photolithography}

Topside, front electrical contact was created onto the-as synthesized PSi surface employing the photolithography and lift-off methodologies according to our recent report [33]. Firstly, spin coating using a layer of photoresist (PR) onto the PSi substrate was carried out, followed by smooth patterning via photolithography. A thin layer of $\mathrm{Au}$ $(50 \mathrm{~nm})$ and $\mathrm{Ti}(10 \mathrm{~nm})$ were deposited onto the patterned top surface of PSi electrodes. A lift-off process was applied to the remaining PR with the defined patterned electrodes.

\subsection{Materials characterization and capacitive}

\section{sensor evaluation}

Surface morphology, pore sizes, and porous silicon layer thickness have been investigated by fieldemission electron scanning microscopy (FESEM, JEOL-6300F, $5 \mathrm{kV}$ ). Elemental mapping analysis has been also obtained using energy dispersive X-ray (EDX) spectroscopy equipped with the above FESEM instrument. The structural and surface properties of the as-fabricated PSi films have been analyzed using X-ray diffractometer (XRD), Bruker AXS D4 Endeavour X-ray diffractometer using $\mathrm{Cu} \mathrm{K} \alpha$ radiation source and the $\mathrm{X}$-ray photoelectron spectroscopy (XPS), a K-ALPHA spectrometer (Thermo Fisher, USA) with a radiation source AlK $\alpha$ for -10 to $1350 \mathrm{eV}$. The sensor performance has been evaluated via the real-time capacitance measurement during the PSi sensor device infiltration with different alcohols, mainly methanol, ethanol, propanol and butanol at the optimum applied frequency using the electrochemical workstation (Zahner Zennium, Germany). The sensing behavior of the currentdeveloped PSi sensor device has been also tested for pure water under the same experimental conditions used for the detection of liquid alcohols.

\section{Results and discussions}

\subsection{Morphological investigation and surface properties of mesoporous silicon}

Fig. 1 shows the FESEM micrographs for the topview (image a) and cross-sectional view (image b) of as-fabricated PSi layer by the photo-electrochemical 
technique using the n-type Si (111) wafer. The topview micrograph of Fig. 1(a) exhibits high-density pore arrays, with randomly distributed manner having mesoporous structure, the pore sizes with the average range of 25-50 $\mathrm{nm}$ (mesoporous regime) can be observed. The formed mesopores are separated from each other with a certain pore-to-pore distance which is not strictly equal over the substrate surface. The cross-sectional view image of Fig. 1(b) reveals that the
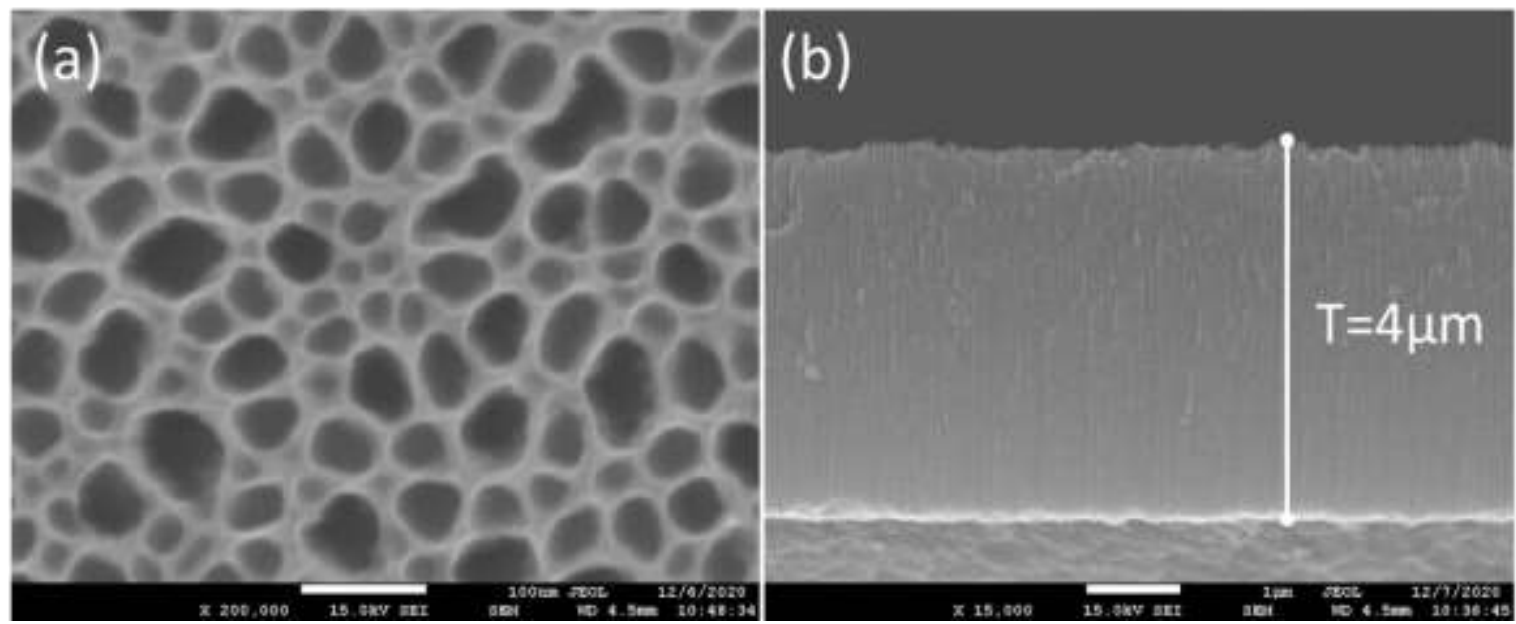

Fig. 1 FESEM images of porous silicon layer fabricated by the photo-electrochemical technique using $n$-type Si (100) substrate; (a) top-view and (b) cross-sectional view.


Fig. 2 EDX elemental mapping analysis for the porous silicon layer fabricated by the photo-electrochemical technique using $n$-type Si (100) substrate; (a) FESEM image, (b) Elemental analysis, (c) Si mapping, and (d) Oxygen mapping. 
Fig. 2 shows the SEM image together with the EDX elemental mapping analysis for the as-fabricated PSi layer. The SEM image (a) again confirmed the presence of high-density and nicely distributed pores over the Si surface. The EDX elemental analysis shown in spectrum (b) clearly confirmed the presence of both $\mathrm{Si}$ and $\mathrm{O}$ elements, with $85.56 \mathrm{wt}$.\% for $\mathrm{Si}$ and 14.44 wt.\% for O. Additionally, the EDX mapping pattern shown in images (c) and (d) revealed the distribution of both $\mathrm{Si}$ and $\mathrm{O}$ elements, respectively. The appearance of oxygen in the as-formed PSi film is likely related to the partial oxidation that occurred during sample storage or transfer.

Fig. 3 shows the XRD patterns of both pristine $\mathrm{Si}$ wafer (with no pores) and the as-synthesized PSi layer. As can be noticed, a sharp and intense diffraction peak appeared at $2 \theta=69.2^{\circ}$ for both PSi and pristine $\mathrm{Si}$, which corresponds to the $\mathrm{Si}(400)$ reflection, inconsistent with the published report [16]. It is worthy to mention also that the crystallinity of the starting silicon wafer was preserved after the porous silicon formation. However, a peak broadening was observed for the PSi sample, suggesting a nonuniform strain distribution with a reduction in crystallite size in the porous layer after the photoelectrochemical anodization process compared to the pristine Si [34].

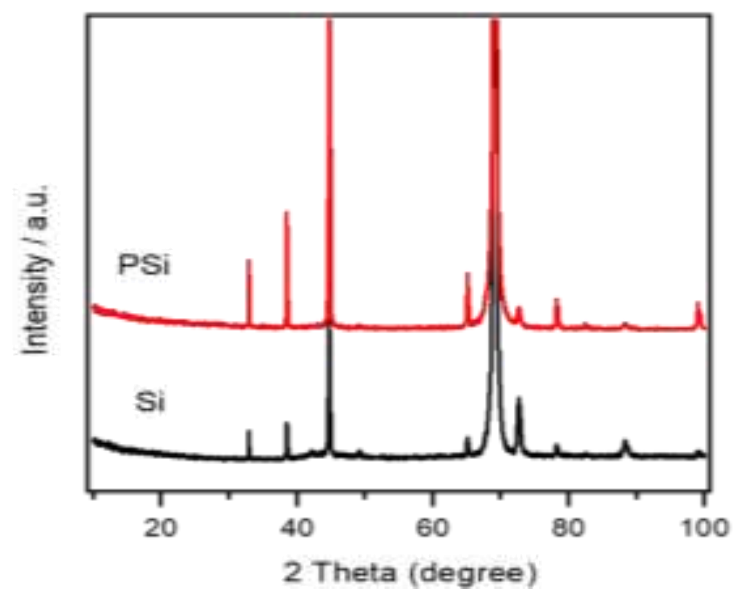

Fig. 3 XRD pattern of porous silicon layer fabricated by the photo-electrochemical technique using $n$-type $\mathrm{Si}$ (100) substrate. The pattern obtained for pristine Si wafer without porosification is also shown as a reference.

The XPS spectral analysis has been done with an ultimate objective to obtain further details on the porous silicon surface chemistry and composition. Fig. 4 illustrates the survey spectrum and the highresolution spectra for both $\mathrm{Si} 2 \mathrm{p}$ and $\mathrm{O} 1 \mathrm{~s}$. As revealed from Fig. 4(a), the surface chemistry of the as- synthesized PSi layer is composed mainly of Si2p and O1s elements, consistent with the above result of EDX elemental analysis of Fig. 2. However, the appearance of C1s spectral peak at a binding energy of $284.6 \mathrm{eV}$ may arise from the rotating pump oil or the environmental carbon. The high-resolution scan of Si2p, Fig. 4(b), shows two separate spectral peaks that appeared at 99.6 and $103.7 \mathrm{eV}$, which can be ascribed respectively to $\left(\mathrm{Si}^{0}\right)$ and $\mathrm{SiO}_{2}[35,36]$. In addition, the core-level spectrum of $\mathrm{O} 1 \mathrm{~s}$ as shown in Fig. 4(b) revealed a single spectral peak at $532.5 \mathrm{eV}$ corresponds to oxide oxygen [37]. As explained above, this oxide formation is likely due to the high tendency of PSi active surface towards oxidation. In the light of material characterization using both EDX elemental analysis and XPS surface analysis, it is now clear that the as-fabricated PSi film is a partially oxidized surface. Such oxidized PSi surface is considered positive and advantageous to passivate the surface and to achieve the required chemical stability of the sensor device, which in turn would help to avoid the sensor device degradation after prolonged use.

\subsection{Real-time capacitance measurements and PSi-Au-Ti sensor evaluation \\ 3.2.1. Capacitive sensing of alcohols}

To properly measure the real-time capacitance of the current sensor, the optimum applied frequency was determined during the impedance study within a wide range of frequency from $0.1 \mathrm{~Hz}$ up to 100,000 $\mathrm{Hz}$, and the optimum value was found to locate around $1 \mathrm{kHz}$ in accordance with our recently published work [31]. Fig. 5 demonstrates the real-time capacitance collected separately during the infiltration of $10 \mu \mathrm{L}$ from different alcohols in liquid phase into the dry PSi-Au-Ti electrical sensor. Fig. 5 (a) shows the capacitance change with time during methanol infiltration. As can be seen, the recorded capacitance was found to rapidly increase just after methanol infiltration (the up-side red arrow indicates the infiltration process). A decline stage for capacitance was observed then increased again until the capacitance value reached its maximum level ( 4.5 $\mathrm{nF})$ at which the PSi-Au-Ti sensor device is expected to be completely impregnated/saturated with liquid methanol molecules. After reaching the maximum level of capacitance, the methanol molecules start to evaporate which is accompanied to the decrease and return of capacitance to the reverse direction of background, dry sensor state.

The evaporation of methanol molecules from the mesoporous structure of current sensor during its 

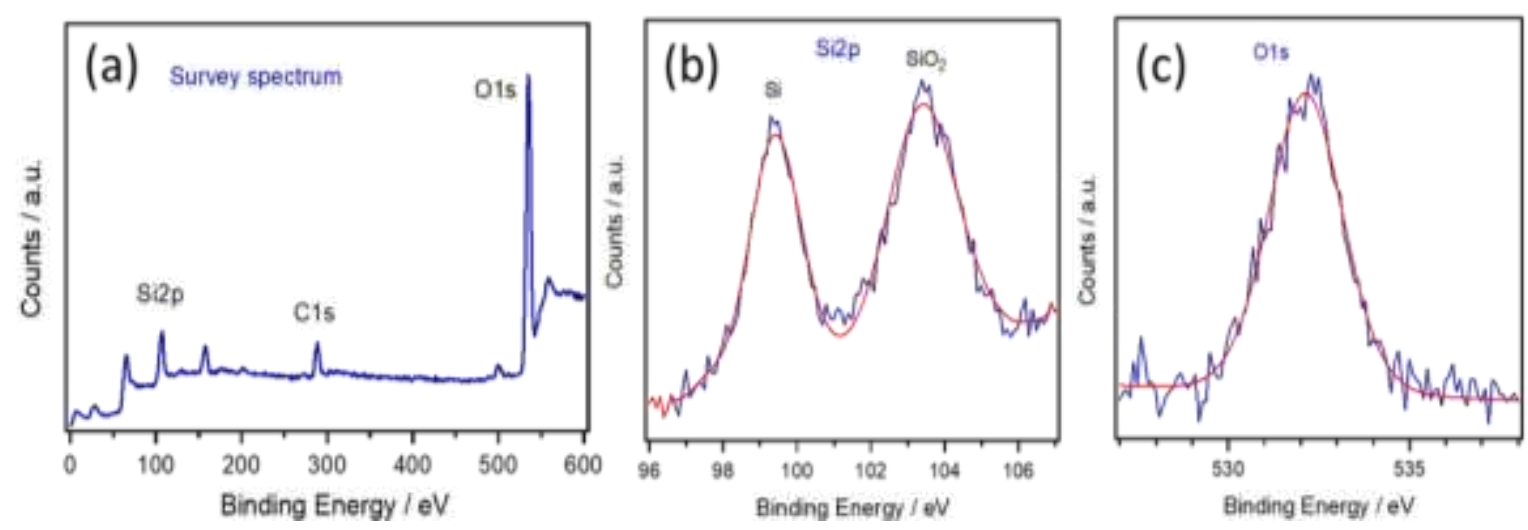

Fig. 4 XPS spectra of porous silicon layer fabricated by the photo-electrochemical technique using $n$-type $\mathrm{Si}$ (100) substrate; (a) Wide scan (survey spectrum) and (b and c) narrow scan (high resolution) spectra measured for Si2p and O1s respectively.

the sensor indicates the reversible behavior of the exposure to methanol, since no chemical reaction or surface changes are expected to occur, which is an advantageous behavior for the current sensor device, as has been also elaborated and addressed by other work [25]. In case of methanol, the response time which covers both infiltration and evaporation stages is around 500 seconds for partially evaporated sensor, and around 1700 seconds for almost complete dry sensor. Fig. 5 (b) shows the real-time capacitance behavior for the case of ethanol. The maximum capacitance value observed in this case was around 2 $\mathrm{nF}$ and the sensor response time for both infiltration and evaporation reached around 3500 seconds, and the partial evaporated state is detected around 1500 seconds. That means the sensing behavior (sensitivity and response time) of the current sensor towards ethanol liquid is much slower compared to methanol. In case of both propanol and butanol, Fig. 5 (c,d), the sensing behavior towards both molecules is completely different from the behavior observed for methanol and ethanol, Fig. 5 (a,b). The maximum capacitance obtained for propanol is only $0.85 \mathrm{nF}$, whereas the value detected for butanol is around 1.6 $\mathrm{nF}$. Additionally, no complete evaporation of either propanol or butanol was observed even after 4000 seconds of operation, and consequently, the sensor did not return to its initial dry state. It is most likely that a surface modification and/or chemical reaction takes place upon the exposure of PSi sensor to either propanol or butanol.

Based on the above sensing behavior, it is clear that the current developed electrical sensor of PSi-Au$\mathrm{Ti}$ is much more appropriate to be implemented to detect simple organic solvents like methanol (one carbon) or ethanol molecule (two carbon chain) as the wetting ability seems to be high which would lead to an enhanced microcapillary condensation force. As the carbon chain increases for propanol (three-carbon structure) and butanol (four-carbon moiety), the wettability is poor and consequently, the capacitive sensing process is quite slow and the reaction seems to be irreversible, which is not appropriate and considered as a negative point for electrical sensor applications.

Now a question arises to understand the reason behind the capacitance change and consequently the sensor response upon the infiltration with organic solvents such as methanol and ethanol liquid. Firstly, we have to keep in mind that the dipole moment effect is one of the important factors for capacitance change. Upon exposure and infiltration of the organic molecules into the mesoporous structure of the sensor, localized electrical field at the interface can be accumulated. This induced local electrical field might cause a shift in the energy level of surface states, which in turn can lead to building a modified spacecharge region (SCR) at the PSi interface [38]. This modified SCR is the main reason to significantly affect the energy distribution [38], which could lead to the observed change in sensor capacitance.

\subsubsection{Capacitive sensing behavior to water}

In this section, we tried to examine the applicability of the current sensor device to detect aqueous solutions and we have taken the pure water as a model. Firstly, the freshly prepared PSi film that is usually terminated by hydrogen and its surface is being passivated was examined. 

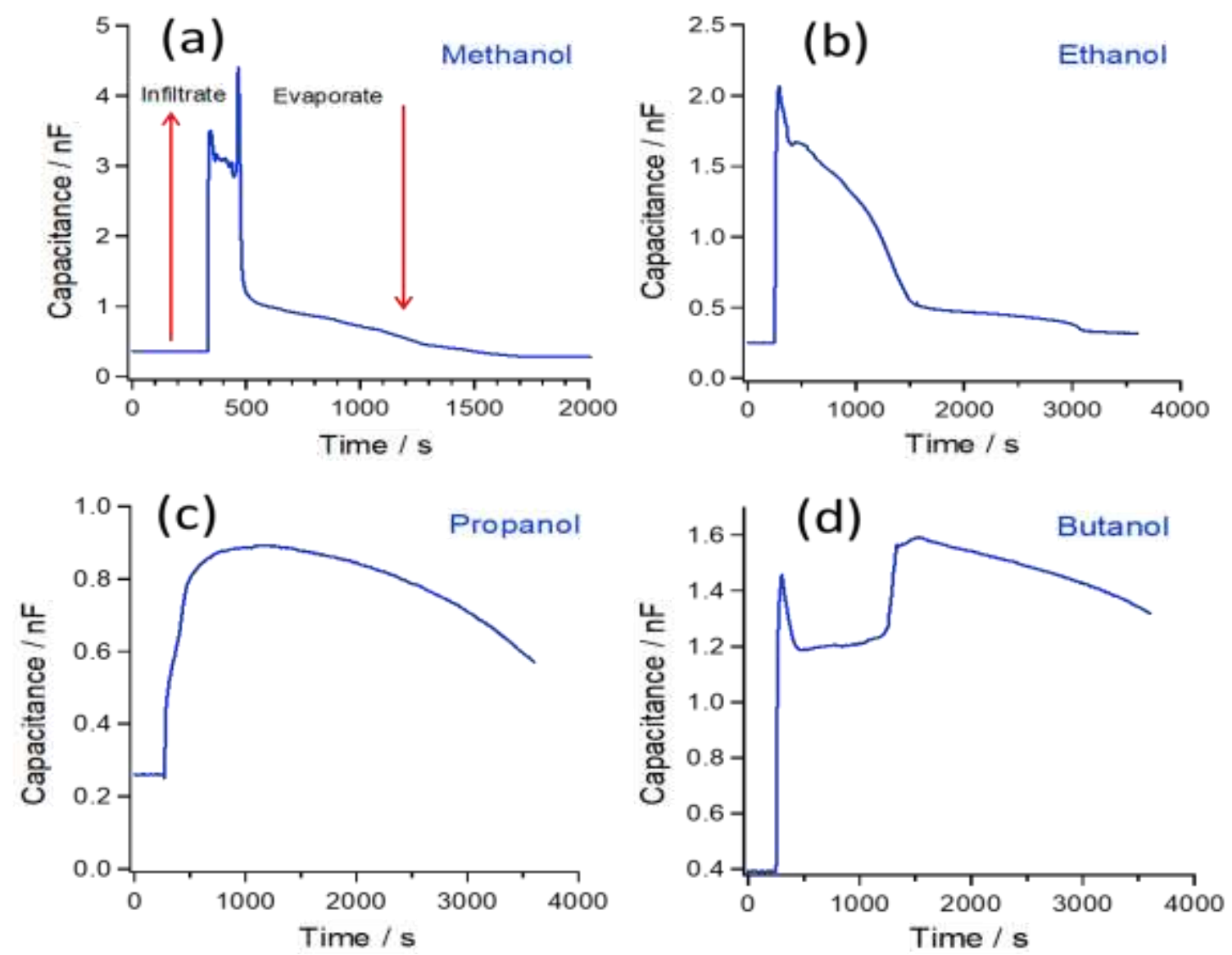

Fig. 5 A comparison of real-time capacitance measurement recorded at applied frequency of $1 \mathrm{kHz}$ and room temperature upon the infiltration with $10 \mu \mathrm{L}$ liquid alcohols into the dry PSi-Au-Ti sensor device; (a) Methanol, (b) Ethanol, (c) Propanol and (d) Butanol.

Sensing results showed that no capacitive response to water was observed, which can be related to the hydrophobic surface character of the freshlysynthesized PSi film. It has been found that water droplets remained without any change onto the front porous silicon surface for prolonged time extending to hours. It is generally accepted and confirmed by experiments that the capacitive response to water could be greatly affected as the result of thermal oxidation of PSi film [25]. It is worthy to mention that the front electrical contact of $\mathrm{Au}-\mathrm{Ti}$ nanocomposite has been created onto PSi surface by lithography and thermal evaporation techniques, and thus the PSi structure is significantly oxidized. The oxidized PSi$\mathrm{Au}-\mathrm{Ti}$ capacitive sensor was examined as a sensing device toward water as shown in Fig. 6. In such a case, it has been observed that the water molecules slowly infiltrated, penetrated the meso-pores of PSi film, leading to a notable wetting of the internal pores, and thereby affecting the capacitance value of the hydrophilic surface of PSi film. As could be noticed in Fig. 6, the sensing response to water was found to proceed via two main stages. The first step is a sharp and very rapid response that occurred immediately upon the first exposure to water droplets, followed by a very slow transition stage represented by a leveling in capacitance response. The second, final stage is accompanied again by an increase in capacitance value until reaching the maximum level of around 1.6 $\mathrm{nF}$. The final stage of response represents a complete wetting of the mesoporous structure by water molecules. A complete reversible cycle of PSi sensor device is detected with a rapid return to the background state representing the initial dry sensor after a total response time of around 2700 seconds, which is considered a rapid response toward water in particular and aqueous solutions in general. Based on the sensing behavior of alcohols and water, it can be concluded that the capacitive sensing behavior of water is more efficient than propanol and butanol, but less efficient if compared to both methanol and ethanol. The sensor sensitivity in terms of capacitance 
change is greatly dependent on various parameters including the vapor pressure and surface tension of examined molecules.



Fig. 6 A real-time capacitance change recorded at applied frequency of $1 \mathrm{kHz}$ and room temperature upon the infiltration with $10 \mu \mathrm{L}$ pure water into the dry PSi-Au-Ti sensor device.

The observed increase of capacitance upon water exposure as represented in Fig. 6 is not strange but expected since water could increase the capacitance [39] and conductance [40] of PSi matrix as has been previously reported. This is actually the prime working principle of humidity sensors based on PSi structures. For example, Anderson et al. [41] have reported the utilization of PSi as a sensing element for humidity sensor for the detection of water vapor. They have explained the capacitance change of PSi sensor by a vapor-condensation mechanism, which led to the change in the effective permittivity of the PSi sensor device. In general, the changes in dielectric constant and also in dipole moment, as well as the possibility of physi-sorption and chemi-sorption into the PSi sensing films have been proposed to explain the capacitive sensing response [42].

However, before reaching a possible commercial use of PSi sensor device, proper solutions from the scientific community for some existing challenges should be elaborated and thoroughly provided. These include innovative device engineering and fabrication of efficient ohmic contacts; proper surface stabilization to minimize the corrosion of the device and enhance the sensor reusability and durability, to fabricate well-ordered pore arrays of PSi to further enhance the device uniformity, to significantly enhance the sensor sensitivity and minimize the fouling behavior of the device due to impurities accumulation, and to propose new approaches that can help increase the sensor's specificity/selectivity which is one of the ultimate objectives of the scientific community for sensor-related technology.

\section{Conclusion}

In summary, we have demonstrated the design and fabrication of a novel, efficient electrical contact of Au-Ti nanostructure created on the front side of meso-PSi film and used successfully for the capacitive sensing of different alcoholic molecules in the liquid phase. The current sensor architecture has provided a significant pathway and effective route to the enhanced capacitive performance towards methanol, ethanol, and pure water in terms of sensitivity, reversibility and rapid response time. The research findings achieved in this work suggest that the creation of an efficient electrical contact at the front side is a promising technique for enhanced electrical sensor related applications. However, further research works are required to achieve rapid response time, more sensitive and selective device with long-term stability and reusability. The near future will show whether the PSi-based sensor device will find its way from research labs into commercial use.

\section{Acknowledgments}

Authors would like to acknowledge the support of the Deputyship for Research and Innovation-Ministry of Education, Kingdom of Saudi Arabia for this research through a grant (NU/IFC/ENT/01/002) under the Institutional Funding Committee at Najran University, Kingdom of Saudi Arabia.

\section{Conflict of interest}

The authors declare no competing interests.

\section{References}

[1] A. Uhlir, Bell Labs Tech. J., Electrolytic Shaping of Germanium and Silicon, 35 (1956) 333.

[2] V. Kumar, Nanosilicon, first ed., Elsevier, 2007.

[3] K. Fukami, F. A. Harraz, T. Yamauchi, T. Sakka, Y. H. Ogata, Fine-tuning in size and surface morphology of rod-shaped polypyrrole using porous silicon as template, Electrochem. Commun. 10 (2008) 56.

[4] Y. H. Ogata, A. Koyama, F. A. Harraz, M. S. Salem, T. Sakka, Electrochemical formation of 
porous silicon with medium-sized pores, Electrochemistry, 75 (3) (2007) 270.

[5] F. A. Harraz, M. S. Salim, T. Sakka, Y. H. Ogata, Hybrid nanostructure of polypyrrole and porous silicon prepared by galvanostatic technique, Electrochim. Acta 53 (2008) 3734.

[6] Y. H. Ogata, J. Sasano, J. Jorne, T. Tsuboi, F. A. Harraz, T. Sakka, Immersion Plating of Copper on Porous Silicon in Various Solutions, Phys. Stat. Sol. (a) 182 (2000) 71.

[7] M. A. Tischler, R. T. Collins, J. H. Stathis, J. C. Tsang, Luminescence degradation in porous silicon, Appl. Phys. Lett. 60 (1992) 639.

[8] A. G. Cullis, L. T. Canham, P. D. Calcott, The structural and luminescence properties of porous silicon, J. Appl. Phys. 82 (1997) 909.

[9] V. Lehmann, The Physics of Macropore Formation in Low Doped n-Type Silicon, J. Electrochem. Soc. 140 (1993) 2836.

[10] E.K. Propst, P.A. Kohl, The electrochemical oxidation of silicon and formation of porous silicon in acetonitrile, J. Electrochem. Soc. 141 (1994) 1006.

[11] V. Lehmann, Electrochemistry of Silicon: Instrumentation, Science, Materials and Applications, Wiley-VCH, Weinheim, 2002.

[12] F.A. Harraz, T. Sakka, Y.H. Ogata, A comparative electrochemical study of iron deposition onto $\mathrm{n}$ - and p-type porous silicon prepared from lightly doped substrates, Electrochim. Acta 50 (2005) 5340.

[13] S. Ottow, V. Lehmann, H. Föll, Processing of Three-Dimensional Microstructures Using Macroporous n-Type Silicon, J. Electrochem. Soc. 143 (1996) 385.

[14] K. Kobayashi, F. A. Harraz, S. Izuo, T. Sakka, Y. H. Ogata, Macropore growth in a prepatterned ptype silicon wafer, Phys. Stat. Sol. (a) 204 (5) (2007) 1321.

[15] K. Imai, A new dielectric isolation method using porous silicon, Solid State Electron. 24 (1981) 159.

[16] J. D. Benjamin, J. M. Keen, A.G. Cullis, B. Innes, N.G Chew, Large area, uniform silicon-oninsulator using a buried layer of oxidized porous silicon, Appl. Phys. Lett. 49 (1986) 716.

[17] R. C. Erson, R. S. Muller, C. W. Tobias, Investigations of porous silicon for vapor sensing, Sensors Actuators A: Physical 23 (1990) 835.

[18] T. Tahercio, M Dilhan, E Massone, A Foucaran, A. M. Gue, T. Bretagnon, B. Fraisse, L. Montes, Porous silicon membranes for gas-sensor applications, Sensors Actuators A: Physical 4647 (1995) 43.

[19] M.S. Salem, M.J. Sailor, F.A. Harraz, T. Sakka, Y.H. Ogata, Electrochemical stabilization of porous silicon multilayers for sensing various chemical compounds, J. Appl. Phys. 100 (8) (2006) 083520.

[20] M.S. Salem, M.J. Sailor, F.A. Harraz, T. Sakka, Y.H. Ogata, Sensing of chemical vapor using a porous multilayer prepared from lightly doped silicon, Phys. Stat. Sol. (c) 4 (6) (2007) 2073.

[21] W. Lang, P. Steiner, A. Richter, K. Marusczyk, G. Weimann, H. Sandmaier, Application of porous silicon as a sacrificial layer, Sensors Actuators A: Physical 43 (1994) 239.

[22] P.C Searson, Porous silicon membranes, Appl. Phys. Lett. 59 (1991) 832.

[23] B. Ünal, A.N. Parbukov, S.C. Bayliss, Opt. Mater. Photovoltaic properties of a novel stain etched porous silicon and its application in photosensitive devices, 17 (1-2) (2001) 79.

[24] L.T. Canham, C.L. Reeves, A. Loni, M.R. Houlton, J.P. Newey, A.J. Simons, T.I. Cox, Calcium phosphate nucleation on porous silicon: factors influencing kinetics in acellular simulated body fluids, Thin Solid Films 297(1-2) (1997) 304.

[25] F.A. Harraz, A.A. Ismail, S.A. Al-Sayari, A. AlHajry, M.S. Al-Assiri, A highly sensitive and durable electrical sensor for liquid ethanol using thermally-oxidized mesoporous silicon, Superlattices Microstruct. 100 (2016) 10641072.

[26] F.A. Harraz, A.A. Ismail, H. Bouzid, S.A. AlSayari, A. Al-Hajry, M.S. Al-Assiri, Electrical porous silicon sensor for detection of various organic molecules in liquid phase, Phys. Status Solidi. 212 (2015) 1851-1857.

[27] F.A. Harraz, A.A. Ismail, H. Bouzid, S.A. AlSayari, A. Al-Hajry, M.S. Al-Assiri, A capacitive chemical sensor based on porous silicon for detection of polar and non-polar organic solvents, Appl. Surf. Sci, 307 (2014) 704-711.

[28] F.A. Harraz, A.A. Ismail, H. Bouzid, S.A. AlSayari, A. Al-Hajry, M.S. Al-Assiri, Mesoporous silicon layer as a highly sensitive ethanol sensor, Int. J. Electrochem. Sci. 9 (2014) 2149-2157

[29] I. Schechter, M. Ben-Chorin, A. Kux, Gas sensing properties of porous silicon, Anal Chem, 67 (1995) 3727. 
[30] M. Yamana, N. Kashiwazaki, A. Kinoshita, T. Nakano, M. Yamamoto, C.W. Walton, J Electrochem Soc, 137 (1990) 2925.

[31] X. G. Zhang, Morphology and Formation Mechanisms of Porous Silicon, J. Electrochem. Soc. 151 (2004) C69.

[32] Farid A. Harraz, Porous silicon chemical sensors and biosensors: A review, Sensors and Actuators B: Chemical, 202 (2014) 897-912.

[33] Md.A. Rashed, Ali S. Alkorbi, Farid A. Harraz, Novel porous silicon/MEH-PPV nanohybrid electrical sensor for sensitive detection of liquid methanol, Materials Letters. 293 (2021) 129734.

[34] A.E. Pap, K. Kordás, G. Tóth, J. Levoska, A. Uusimäki, J. Vähäkangas, S. Leppävuori, T.F. George, Thermal oxidation of porous silicon: Study on structure, Appl. Phys. Lett. 86 (2005) 41501.

[35] F. A. Harraz, Synthesis and surface properties of magnetite $\left(\mathrm{Fe}_{3} \mathrm{O}_{4}\right)$ nanoparticles infiltrated into porous silicon template, Applied Surface Science 2013, 287, 203-210.

[36] F. A. Harraz, J. Sasano, T. Sakka, Y. H. Ogata, Different Behavior in Immersion Plating of Nickel on Porous Silicon from Acidic and Alkaline Fluoride Media, J. Electrochem. Soc. 2003, 150, C277-C284.

[37] F. González, E. Barrera-Calva, L. Huerta, R. S. Mane, Coatings of $\mathrm{Fe}_{3} \mathrm{O}_{4}$ Nanoparticles as Selective Solar Absorber, The Open Surf. Sci. J. 2011, 3, 131-135.

[38] I. Schechter, M. Ben-Chorin, A. Kux, Gas Sensing Properties of Porous Silicon, Anal. Chem., 67 (20) (1995) 3727-3732.

[39] Z.M. Rittersma, A. Splinter, A. Boedecker, W. Benecke, A novel surface-micromachined capacitive porous silicon humidity sensor, Sens. Actuat. B 68 (2002) 210-217.

[40] F. Moeller, M. Ben-Chorin, F. Koch, Posttreatment effects on electrical conduction in porous silicon, Thin Solid Films 255 (1995) 1619.

[41] R.C. Anderson, R.S. Muller, C.W. Tobias, Investigation of porous silicon for vapor sensing, Sens. Actuat. A, A21/A23 (1990) 835-839.

[42] M. Archer, M. Christophersen, P. M. Fauchet, Electrical porous silicon chemical sensor for detection of organic solvents, Sensor Actuator B 106 (2005) 347-357. 